\title{
Effect of image characteristics of edge-grain patterns on visual impressions
}

$\operatorname{AUTHOR}(\mathrm{S})$ :

Nakamura, Masashi; Miyake, Yumi; Nakano, Takato

\section{CITATION:}

Nakamura, Masashi ... [et al]. Effect of image characteristics of edge-grain patterns on visual impressions. Journal of Wood Science 2012, 58(6): 505-512

ISSUE DATE:

2012-12

URL:

http://hdl.handle.net/2433/168516

\section{RIGHT:}

The final publication is available at www.springerlink.com; この論文は 出版社版でありません。引用の際には出版社版をご確認ご利用くださ $\omega_{\circ}$; This is not the published version. Please cite only the published version. 
Title

Effect of image characteristics of edge-grain patterns on visual impressions

\section{Type of article}

5 Original article

\section{Authors}

Masashi Nakamura*, Yumi Miyake, Takato Nakano

Graduate School of Agriculture, Kyoto University,

10 Kitashirakawa Oiwake-cho, Sakyo-ku, Kyoto 606-8502, Japan

E-mail: nakamasa@kais.kyoto-u.ac.jp

\section{Keywords}

Edge-grain pattern, Multi-resolution contrast analysis, Sensory evaluation, Visual impression 


\begin{abstract}
To demonstrate the psychophysical effect of edge-grain patterns on visual impressions received by human observers, printed images of the edge-grain patterns of 16 species were prepared as wellregulated visual stimuli, and impressions of the patterns on 32 subjects were evaluated using six

5 categories. Image characteristics of the patterns were expressed numerically using contrast values that were derived by multi-resolution contrast analysis (MRCA) proposed by the authors, and relationships between the image characteristics and the visual impressions were examined. The difference in lightness between local areas with a certain size in the image was used as the contrast value, which could be used to detect the change in lightness due to the particular feature in the edge-grain

10 pattern. While some impressions were almost independent of the contrast values, other impressions showed highly positive or negative linear relationships to the contrast values, derived at the optimal filter size in MRCA. Moreover, these optimal sizes were rarely affected by color information. The contrast value proposed in this paper would be a good numerical index that could be used not only to describe image characteristics of the edge-grain patterns but also to estimate some of their visual
\end{abstract} 15 impressions. 


\section{Introduction}

The unique appearance of wood, which is characterized by warm colors, varied grain patterns, mellow gloss, and so on, has various psychological effects on observers. Nyrud and Bringslimark [1] pointed out in their review article that the affective responses of human observers to wood provided

5 psychologically beneficial effects. To determine more precisely the nature of these desirable effects, relationships between the surface properties of wood and visual impressions of the surfaces have been examined. In some reports [2-6], the surface properties were assessed on the basis of subjective evaluations by human graders, the visual impressions were reported by human observers, and the relationships between them were investigated using various multivariate analyses. In other reports [7-13], the surface properties were converted into numerical characteristics by image analyses and were compared with the visual impressions that were evaluated subjectively. However, there have not been many systematic investigations considering those properties of the surfaces that brought about visual effects. In other studies, the authors developed a multi-resolution contrast analysis (MRCA) to describe the change in the appearance of lumber surfaces numerically [14,

15 15]. MRCA employs a computation algorithm that considers the human vision system and derives characteristic values that correspond to the surface attributes. However, our verification of how well the image characteristics derived by MRCA correspond with the visual impressions has not been satisfactory.

The objective of the present study is to demonstrate the psychophysical effects of the grain pat-

20 terns on the visual impressions received by human observers. To approach this issue as systematically as possible, it is necessary that the shape, color, and gloss of the grain patterns be controlled by using prepared photographic specimens. To simplify the shape-related problem, edge-grain patterns were preferred because these were considered as relatively simple stripes. To reduce the colorrelated problem, all patterns were converted to digital images, and their color attributes were regulated by image processing. To avoid the gloss-related problem, all images were printed on the same 
photographic paper. Image characteristics of the edge-grain patterns were calculated by MRCA, and the visual impressions of the patterns were assessed by sensory evaluation.

\section{Materials and methods}

5 Specimens

To prepare various kinds of the edge-grain pattern as much as possible, planer-finished quartersawn boards of 16 tree species were collected in consideration of their anatomical features (softwood or hardwood, ring porous or diffuse porous, and so on), annual ring density, and homogeneity of the grain. The boards were approximately 150,100, and $5 \mathrm{~mm}$ in the longitudinal, radial, and tangential directions, respectively. Digital color images of 16 boards were captured by a flatbed scanner (CanoScan9950F, Canon Inc., Tokyo, Japan) with high resolution（1200 dots per inch; 7000 pixels $\times 4600$ pixels).

Using the captured images, three types of printed specimens were prepared as the visual stimuli to be presented in sensory evaluation. All specimens were printed by an ink-jet printer (PIXUS

15 860, Canon Inc., Tokyo, Japan) on matte printing paper（SG-101, Canon Inc., Tokyo, Japan) with the same resolution and size $(1200 \mathrm{dpi}, 148 \mathrm{~mm} \times 97 \mathrm{~mm})$ through photo-retouching software (Photoshop CS1, Adobe Systems Inc., Tokyo, Japan).

Type 1 specimens were printed in their original colors. Type 2 specimens were prepared as the monochrome version of Type 1 (Figure 1). The color data (three gray levels of the RGB color sys20 tem) of every pixel in the original color images were converted to a single gray level $g$ by Eq. 1 [16].

$$
g=0.299 R+0.587 G+0.114 B
$$

To convert a gray level $g$ to a metric lightness $L^{*}$ in the $\mathrm{L}^{*} \mathrm{a}^{*} \mathrm{~b}^{*}$ color system, the following quintic regression equation was defined.

$$
L^{*}=-466.5 g^{5}+1381 g^{4}-1545 g^{3}+755.5 g^{2}-40.44 g+7.804 .
$$


In Eq. 2, $g$ was standardized from 0 to 1. To define Eq. 2, 19 types of monochrome chips with various intensities were printed out using the previously described process used to print Type 1 and Type 2 images, and their metric lightness values were measured by a colorimeter (CM-2600d, Konica Minolta Optics, Inc. Tokyo, Japan). The non-linear relationships between $g$ and $L^{*}$ were

5 formulated as Eq. 2. Next every monochrome image was transformed to a statistically equivalent image with the following two equations.

$$
\begin{aligned}
& Z(i, j)=\frac{S(i, j)-\bar{S}}{\sigma_{S}}, \\
& D(i, j)=\sigma_{D} Z(i, j)+\bar{D},
\end{aligned}
$$

where $S$ is the source image, $\bar{S}$ indicates the lightness of $S, \sigma_{S}$ is the standard deviation of $S, Z$ is the intermediate image, $D$ is the destination image, $\bar{D}$ indicates the lightness of $D, \sigma_{D}$ is the standard deviation of $D$, and $i$ and $j$ are the horizontal and vertical positions of each pixel. $\bar{D}$ and $\sigma_{D}$ of Type 2 specimens were set to 70.1 and 4.7, respectively. In other words, all Type 2 specimens had the same mean lightness values and standard deviations. The values for Type 2 specimens are tabulated in Fig. 1.

15 In addition, monochrome images of 16 specimens were created, which were named Type 3 specimens. For these specimens, four species in Type 1 or 2, i.e., Nos. 2, 5, 7, and 14 (see Fig. 1) were selected. The variation in lightness of these specimens was modified in four ranges by changing $\sigma_{D}$ in Eq. 4. In the present study, the mean lightness of $\bar{D}$ in Eq. 4 was set to 70.1, as it was for Type 2 specimens, and $\sigma_{D}$ values were set to 2.3, 4.7 (same as the values for Type 2 specimens), 7.2, or 9.7. The value of $\sigma_{D}$ corresponds to the magnitude of the overall contrast of the pattern. A part of the Type 3 specimens are shown in Fig. 1. A schematic flow of image processing is shown in Fig. 2. The image processing was performed by self-made FORTRAN programs. 
Sensory evaluations were performed concerning the visual impressions of the edge-grain patterns. A questionnaire asked the subjects to rank their impressions using six adjectives: Close, Decent, Clear, Varied, Showy, and Elegant. The words were translated into corresponding Japanese on the questionnaire form, that is, Chimitsu-na, Totonotta, Hakkiri-shita, Henkani-tonda, Hade-na, and

5 Johin-na, respectively. These adjectives had been used in a former study [17] that measured the visual impressions of wood-based materials. In the present study, the six adjectives were reselected in consideration of the correspondence between the impressions and characteristics of the edge-grain patterns (annual ring density, difference of brightness between earlywood and latewood, homogeneity of the grain, and so on), and also ease of reply by the subjects. In our study, each subject was asked to observe a set of the specimens (Type 1,2, or 3), which were randomly arranged on gray paper (N5 in Munsell value) covering the table, and to sort them in order of the degree of each impression. It took approximately 80 minutes to complete a series of tasks including some rests. The table was illuminated by neutral white fluorescent lamps from the ceiling, and surface illuminance over the table was approximately 1100 lx. Thirty-two students (16 male and 16 female; mean age

$1523.7 \pm 2.0 ）$ participated in answering the questionnaire. They observed the specimens from approximately a distance of $800 \mathrm{~mm}$. Mean visual acuity of the subjects (including users of glasses or the contact lens) was $0.96 \pm 0.28$. Sensory data obtained by the ranking method were statistically scaled, based on the mean standard deviation [18].

Nakamura et al. proposed multi-resolution contrast analysis (MRCA) $[14,15]-$ a new method to analyze the images. In this method, differences of lightness between contiguous areas with a given size are calculated in various places within an image by changing the size of the areas being measured. The idea to compare the lightness between adjacent areas in the image is similar to the retinex theory proposed by Land and McCann when considering the human visual system [19, 20]. In addi- 
tion, the multi-resolution concept in MRCA provides a type of frequency analysis similar to Fourier analysis.

In our study, we tried to characterize the appearance of edge-grain patterns using MRCA. Although Nakamura et al. formulated an algorithm of a two-dimensional MRCA and applied it to various lumber-surface images $[14,15]$, we modified this algorithm to a one-dimensional version and applied it to the edge-grain patterns because these patterns were considered as relatively simple stripes.

First, from each pattern, 16 line profiles perpendicular to the grain were sampled with the same interval. The line profile, $L_{j}(i) \quad(1 \leq i \leq m, 1 \leq j \leq N)$, was a series of lightness changes in the horizontal direction of the pattern, where $i$ denotes the position of a pixel, $j$ denotes a line number, and $m$ and $N$ were the number of pixels composing a line and the number of lines, respectively (in the present study, $m=4096, N=16)$.

The one-dimensional MRCA was applied to each line profile. The procedure was formulated as follows. A smoothing filter was applied to the $j$ th profile,

$$
\bar{L}_{j k}(I)=\frac{1}{k} \sum_{i=(I-1) k+1}^{I k} L_{j}(i)
$$

where $k$ is the filter size denoted by the number of pixels, $I$ is the position of the filtered area $(1 \leq I \leq m / k)$, and $\bar{L}$ indicates the lightness of the area. The Ith local contrast value in the $j$ th smoothed profile was defined by Eq. 6,

$$
C_{j k}(I)=\left|\bar{L}_{j k}(I)-\bar{L}_{j k}(I+1)\right| \quad(1 \leq I \leq m / k-1) .
$$

20 The mean contrast value of the $j$ th profile $\bar{C}_{j k}$ and the whole mean contrast value $C_{k}$ were calculated by Eqs. 7 and 8, respectively,

$$
\bar{C}_{j k}=\frac{1}{m / k-1} \sum_{I=1}^{m / k-1} C_{j k}(I)
$$




$$
C_{k}=\frac{1}{N} \sum_{j=1}^{N} \bar{C}_{j k} .
$$

These calculations were performed by the self-made FORTRAN programs.

The relationship between the filter size $k$ and the mean contrast value $C_{k}$ was expressed as a contrast spectrum. Sixteen contrast spectra of Type 3 specimens are shown in Fig. 3. Each contrast spectrum was a characteristic curve of the edge-grain pattern. In Fig. 3, levels of the contrast spectra improve with an increase in the standard deviation of lightness of each specimen, from lower a to upper $\mathrm{d}$. This means that MRCA could sharply detect lightness changes in any local area. Additionally, for example, the filter size of a peak position in the spectrum of specimen No. 2, which is approximately $1 \mathrm{~mm}$, corresponds to the mean width of the dark stripes of the zebra wood.

\section{Results and discussion}

Correlation coefficients between six impressions in the Type 3 specimens are summarized in Table 1. The Clear, Varied, and Showy impressions were in a highly positive correlation to each other. The Elegant impression showed a highly negative correlation to these three impressions. Although

15 the Close and Decent impressions showed a highly positive correlation to each other, the correlations with the other four impressions were relatively low. Based on this analysis, we classified the six impressions into three groups and demonstrate the relationships between contrast values and impressions regarding the three impressions of Varied, Elegant, and Close.

In Type 3 specimens, an increase in the standard deviation of lightness expanded the range of

20 lightness variations and increased the overall contrast of the edge-grain pattern. The relationships between standard deviations and the three impressions are shown in Fig. 4. The Varied impression increased with an increase in standard deviation of lightness, and conversely, the Elegant impression was on the decline. However, the assessment of each specimen was conducted separately for each species, even if there were similar standard deviations among them. Also, the Close impres- 
sion was assessed for each species independently of the standard deviation.

MRCA derives an inherent contrast value for each filter size. A large filter can detect lightness changes that appear in large areas, and a small filter deals with the changes in smaller areas. The magnitude of the contrast value denotes how noticeable a specific feature is when matched with a

5 given filter size. Using the above properties of MRCA, we examined the correlation between the visual evaluations of an impression and the contrast values at the specific filter size, and we obtained a correlation spectrum that is composed of successively calculated correlation coefficients. The three correlation spectra of the Varied, Elegant, and Close impressions are shown in Fig. 5. The Varied and Elegant impressions presented highly positive and negative correlations when the filter sizes were 1-3 $\mathrm{mm}$ and 1-2 $\mathrm{mm}$, respectively. Though the Close impression had the highest negative and positive correlations when the filter sizes were 1.7 and $17 \mathrm{~mm}$, respectively, their levels were relatively low.

These correlation spectra suggest the existence of an optimum filter size for each impression, which will match the size of a useful visual clue to assess the impression. In the present study,

15 based on the positions of a peak or a valley of the spectra, we selected the following filter sizes as optimum: 2.9, 1.3, and $1.7 \mathrm{~mm}$ for the Varied, Elegant, and Close impressions, respectively. The relationships between the impressions and the contrast values found at each optimum filter size are shown in Fig. 6. The very high correlation for the Varied impression suggests that lightness changes appearing in relatively broad features of the edge-grain patterns affected the impression. Similarly,

20 the lightness changes in relatively narrow features should affect the Elegant impression. However, the Close impression is almost independent of the contrast. Probably, this impression was slightly affected by visual factors of the annual ring density and other factors.

Based on the results from the Type 3 specimens, we attempted to explain the relationship between the contrast values and the two visual impressions in the Type 1 and 2 specimens. Figs. 7 and 258 represent the relationships between the contrast values and the Varied and Elegant impressions in 
Type 1 and 2 specimens, respectively. Although the contrast values of the Type 1 and 2 specimens were determined on the basis of optimal filter sizes specified for Type 3, there were relatively high correlations between the contrast values and the two impressions. Therefore, a particular feature when matched with the filter size was an effective clue to evaluate the impression, and this clue

5 was rarely influenced by the presence of color.

In Fig. 9, the impressions of Varied, Elegant, and Close of the Type 1 specimens are compared with the same impressions of Type 2 specimens. In all three impressions, positive correlations between Type 1 and 2 specimens exist. However, for the Varied and Elegant impressions, a few specimens that were equally evaluated within Type 1 were rated differently within Type 2 , and vice versa. When the colored edge-grain patterns lost their color information and were set to gray tones (that is, conversion from Type 1 to Type 2, as shown in Figure 2), light and shade in the patterns were emphasized or weakened. Probably, such an appearance change resulted in difference in the sensory evaluations between Type 1 and 2 specimens. On the other hand, this clear linear relationship between both sets of specimens means that the Close impression was difficult to be influenced

15 by the changes in color and lightness.

\section{Conclusions}

To demonstrate the psychophysical effects of edge-grain patterns on visual impressions received by human observers, we used sensory evaluation to assess the visual impressions of various edge-grain

20 patterns by using printed images, which were well-regulated by image processing. We computed contrast values as the image characteristics of each pattern on the basis of a one-dimensional MRCA and determined the optimal filter size in MRCA that was congenial to each impression. Although a few impressions such as Close were almost independent of the contrast values, the contrast values and other impressions, such as Varied and Elegant, were highly correlated (positively or negatively). In addition, these relations were robust, and they were rarely influenced by the color 
information. The experimental results demonstrate that our proposed contrast value can provide a good numerical index that not only expresses image characteristics of the edge-grain patterns but also estimates some of their visual impressions.

However, the present study elucidates only a part of the psychophysical properties of the edge-

5 grain patterns based on MRCA. We should examine the versatility of MRCA more widely, and make the methodology more sophisticated to numerically describe the subjective preference or esthetics for wood and wood products. 


\section{References}

1. Nyrud AQ, Bringslimark T (2010) Is interior wood use psychologically beneficial? A review of psychological response toward wood. Wood and Fiber Sci 42: 202-218

2. Broman NO (1995) Attitudes toward Scots pine wood surfaces: A multivariate approach. Mokuzai Gakkaishi 41: 994-1005

3. Broman NO (1996) Two methods for measuring people's preferences for Scots pine wood surfaces: A comparative multivariate analysis. Mokuzai Gakkaishi 42: 130-139

4. Broman NO (2001) Aesthetic properties in knotty wood surfaces and their connection with people's preferences. J Wood Sci 47: 192-198

5. Nyrud AQ, Roos A, Rødbotten M (2008) Product attributes affecting consumer preference for residential deck materials. Can. J. of Forest Research 38: 1385-1396

6. Høbiø O, Nyrud AQ (2010) Consumer perception of wood surfaces: the relationship between stated preferences and visual homogeneity. J. Wood Sci 56: 276-283

7. Masuda M (1983) Studies on numerization of wood pattern and other pattern: especially on numerization of patterns based on flicker (in Japanese). J Soc Mat Sci Japan 32: 893-898

8. Okajima T, Kubo T, Noda K, Fujibayashi K. (1985) A spectral analysis of stripes and its application to visual effects of architectural stripe patterns (in Japanese). J Structural and Construction Engineering -Trans Architectural Institute of Japan 356: 16-23

9. Okajima T, Wakabayashi S, Noda K, Konishi T (1986) A geometrical analysis of the grain of wood and generating its patterns (in Japanese). J Structural and Construction Engineering Trans Architectural Institute of Japan 369: 9-15

10. Okajima T, Wakayama S, Noda K, Konishi T (1987) Grain pattern and its psychological effect (in Japanese). J Structural and Construction Engineering -Trans Architectural Institute of Japan 377: $1-7$ 
Japanese). Mokuzai Gakkaishi 41: 301-308

12. Nakamura M, Masuda M, Imamichi K (1996) Description of visual characteristics of wood influencing some psychological images (in Japanese). Mokuzai Gakkaishi 42: 1177-1187

13. Nakamura M, Sakai T, Masuda M (2002) Visual characteristics influencing visual hardness of wood (in Japanese). J Soc Mat Sci Japan 51: 398-403

14. Nakamura M, Masuda M, Shinohara K (1998) Multiresolutional image analysis of wood and other materials. J. Wood Sci 45: 10-18

15. Nakamura M, Matsuo M, Nakano T (2010) Determination of the change in appearance of lumber surfaces illuminated from various directions. Holzforschung 64: 251-257

16. Motoki N (1998) Principle of Color Television System. In: The Color Science Association of Japan (ed) Handbook of color Science [Second edition] (in Japanese). University of Tokyo Press, Tokyo, pp 986-995

17. Yasuda A, Masuda M, Maku T (1978) Studies on the visual characteristics of wood based materials -Factor analysis on the visual image of the interior wall materials- (in Japanese). Wood Research Review 12: 81-101

18. Nakamura M, Masuda M (1995) Simplified method for statistical rating of sensory data (in Japanese). Wood Industry 50: 18-21

19. Land EH, McCann, JJ (1971) Lightness and retinex theory. J opt Soc Am 61: 1-11

20. Land EH (1977) The retinex theory of color vision. Scientific American 237: 108-128 


\section{Captions for figures}

Fig. 1 Specimen images of edge-grain patterns. The mean and standard deviation of lightness of the Type 2 specimens were equalized to 70.1 and 4.7, respectively. The images were arranged in the order of Varied impression from upper left to lower right. Four species in Type 2 (No. 2, 5, 7 and 14) were selected for Type 3 specimens. Although their mean lightness was the same as that of Type 2, their standard deviations were modulated in four variations. Numbers above and below each image indicate specimen code, mean lightness, and standard deviation, respectively. The sizes of the specimens are $148 \mathrm{~mm}(\mathrm{~L})$ and $97 \mathrm{~mm}(\mathrm{R})$.

10

Fig. 2 Schematic flow of image processing. Left: original color images (Type 1) have various histograms of lightness. Middle: original color images were converted to monochrome images (Type 2), which have same mean and standard deviation of lightness (i.e., statistically equal images). Left: standard deviations of part of the Type 2 specimens were modulated in four variations.

15

Fig. 3 Contrast spectra of Type 3 specimens. The number at the upper left of each graph corresponds to the specimen code of Type 2 specimens, as shown in Figure 1. Spectrum letters (from a to d) denote the variation of standard deviations assigned to Type 3 specimens.

20 Fig. 4 Relationships between standard deviations of lightness and visual impressions in Type 3 specimens. Letters above each graph correspond to the spectrum numbers in Fig. 3. 
Fig. 5 Correlation coefficient spectra of Varied, Close, and Elegant impressions in Type 3 specimens.

Fig. 6 Relationships between contrast values and visual impressions in Type 3 specimens. A meas5 urement enclosed in parentheses is the optimal filter size. $(* p<0.05$, *** $p<0.001)$

Fig. 7 Relationships between contrast values and visual impressions in Type 1 specimens. Contrast values are determined by the optimal filter sizes specified for Type 3. $(* * * p<0.001)$

10 Fig. 8 Relationships between contrast values and visual impressions in Type 2 specimens. Contrast values are determined by the optimal filter sizes specified for Type 3. $(* * * p<0.001)$

Fig. 9 Reciprocal relationships of impressions between Type 1 and 2 specimens. (** $p<0.01$, *** $p<0.001)$ 


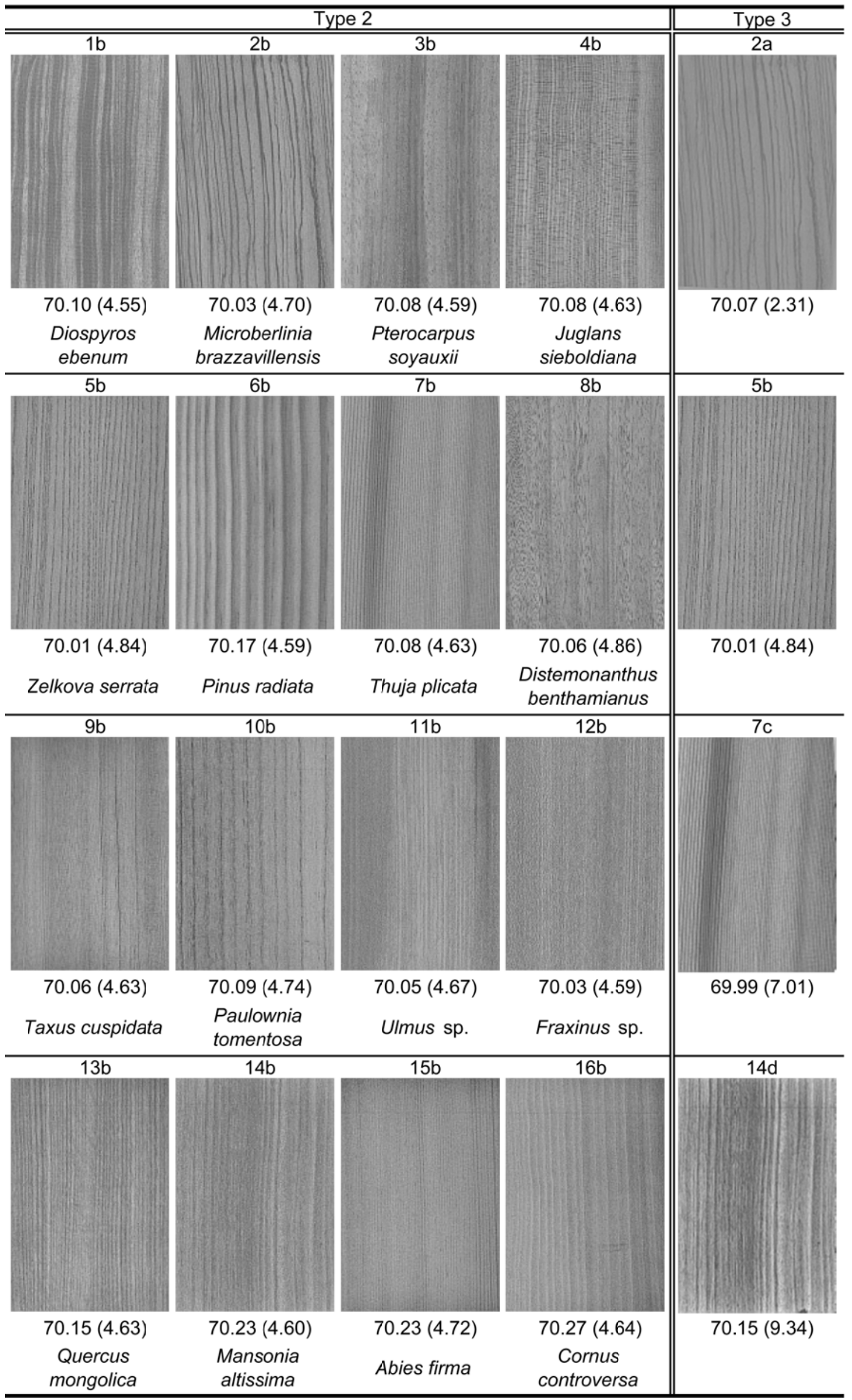

Fig. 1 


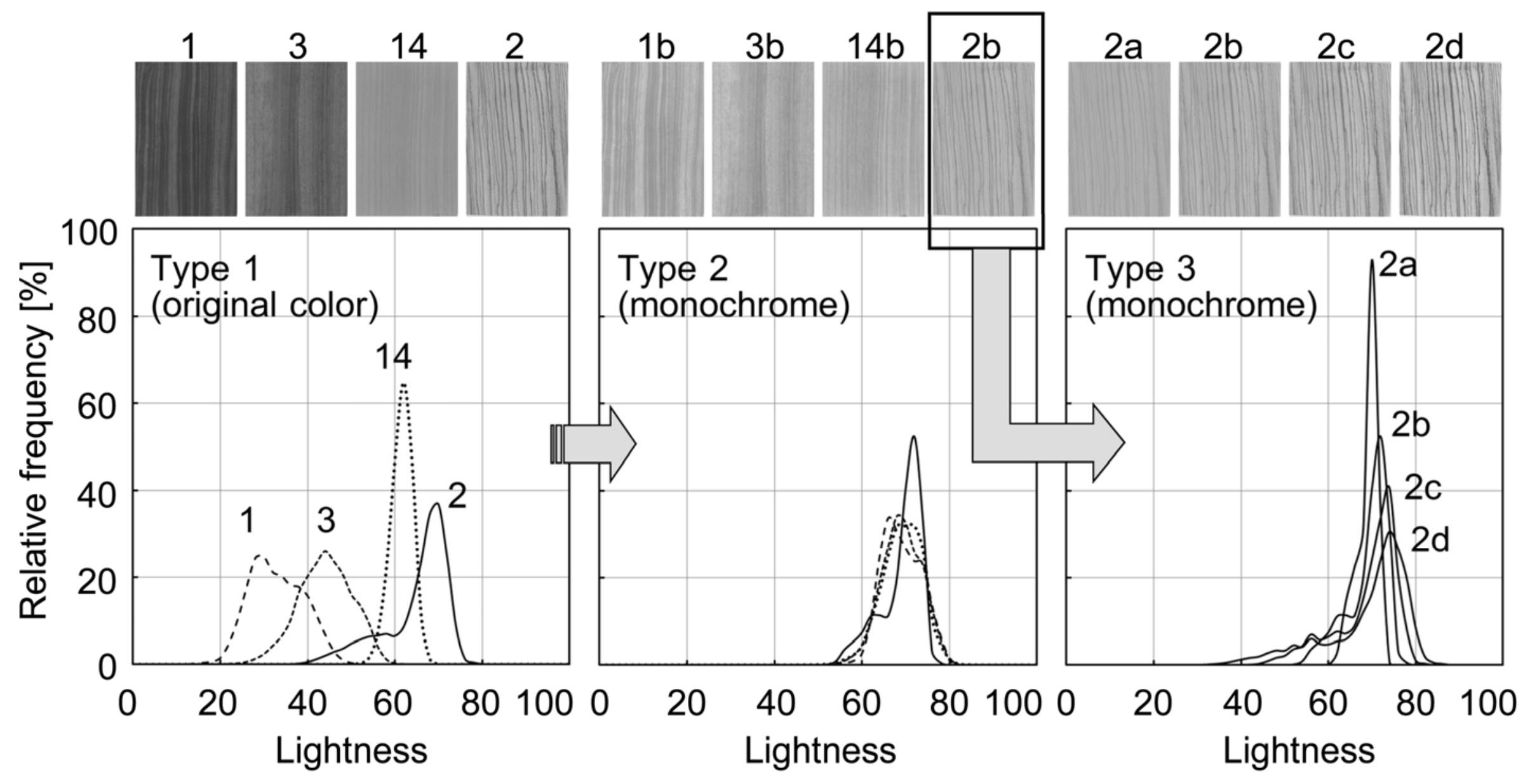

Fig. 2 


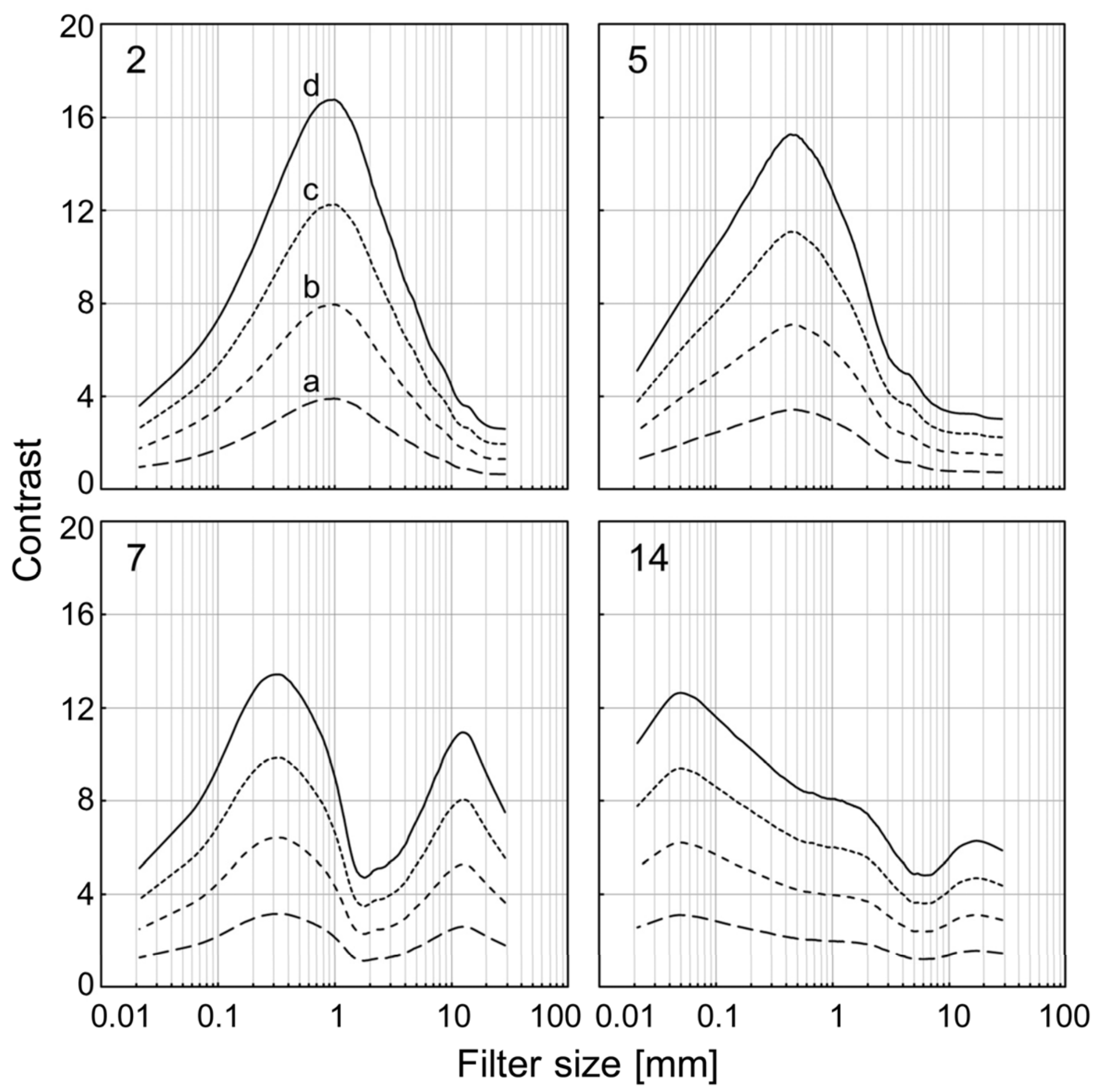

Fig. 3 


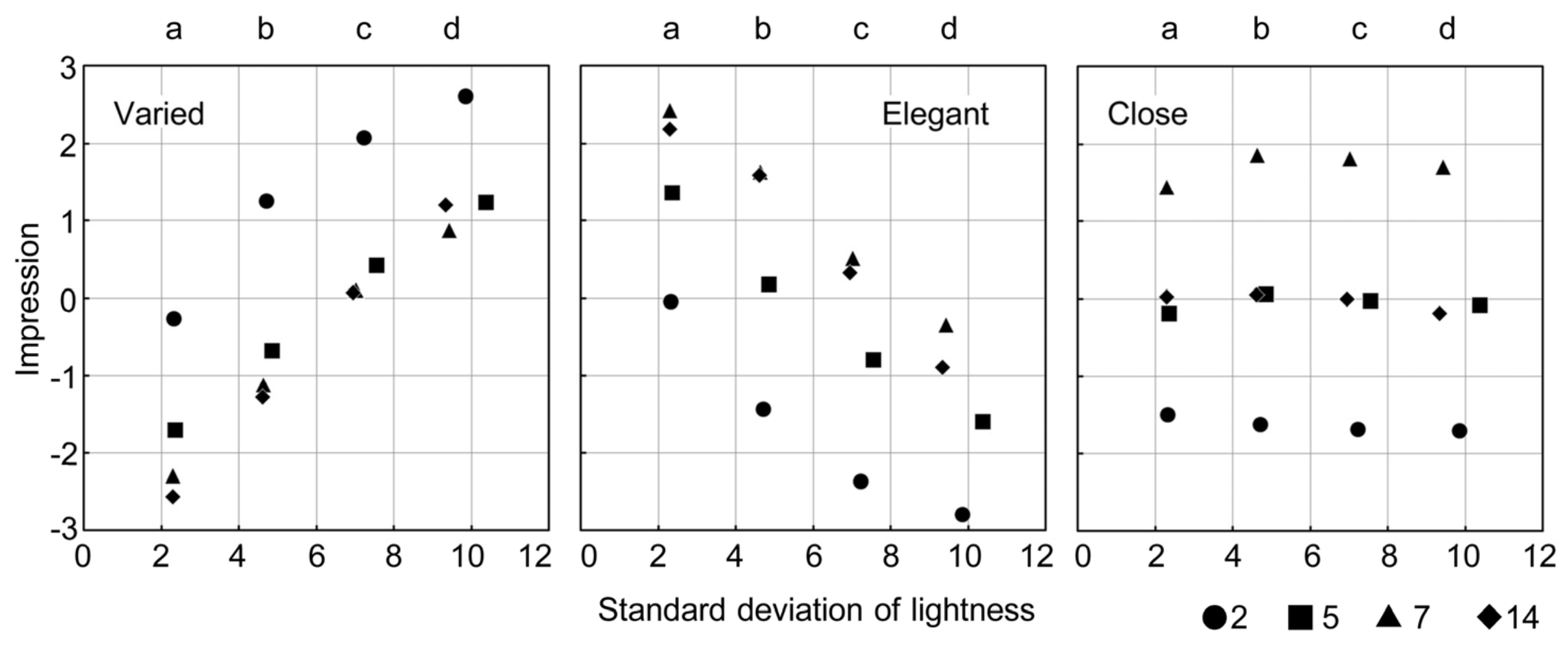

Fig. 4 


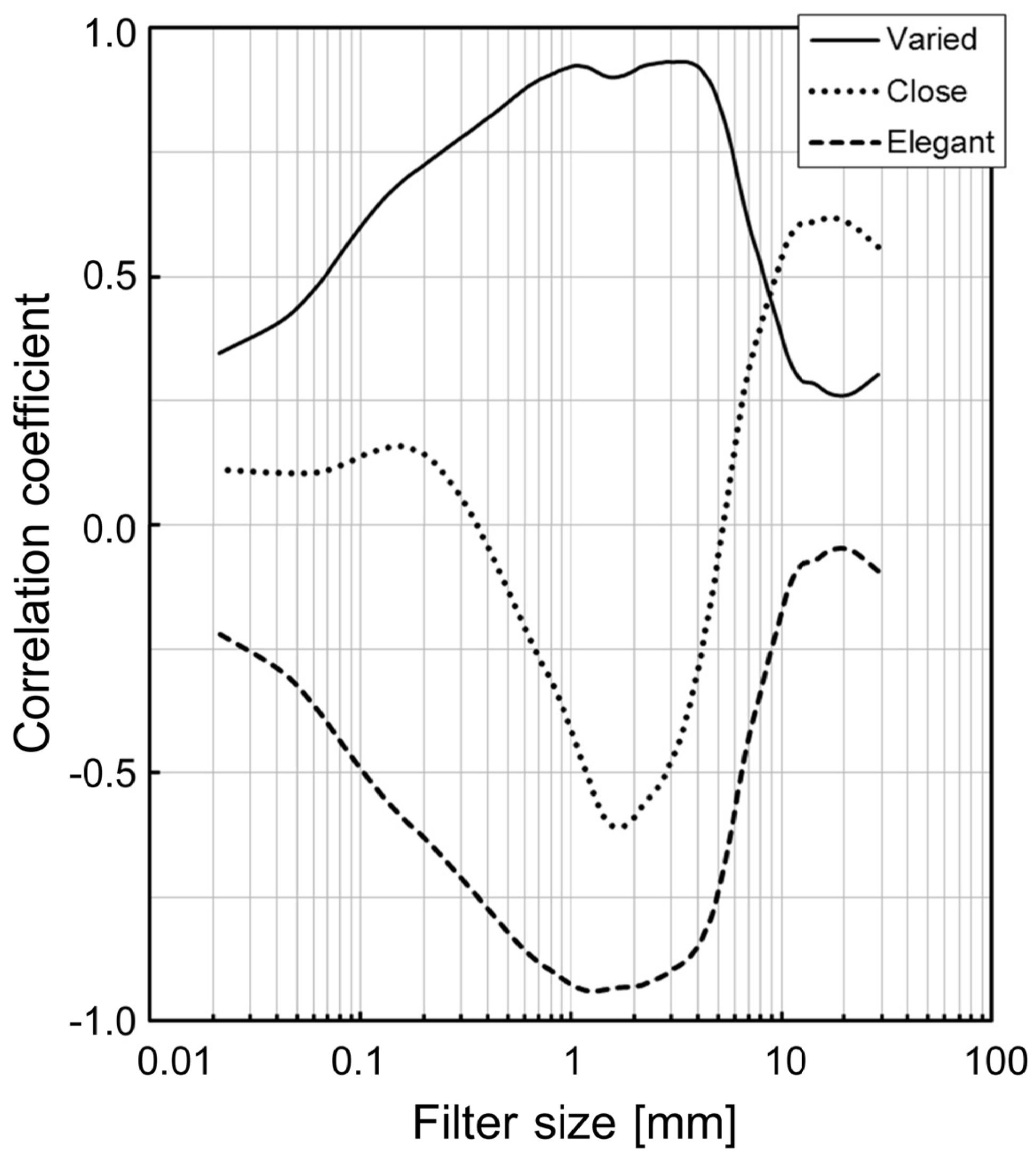

Fig. 5 

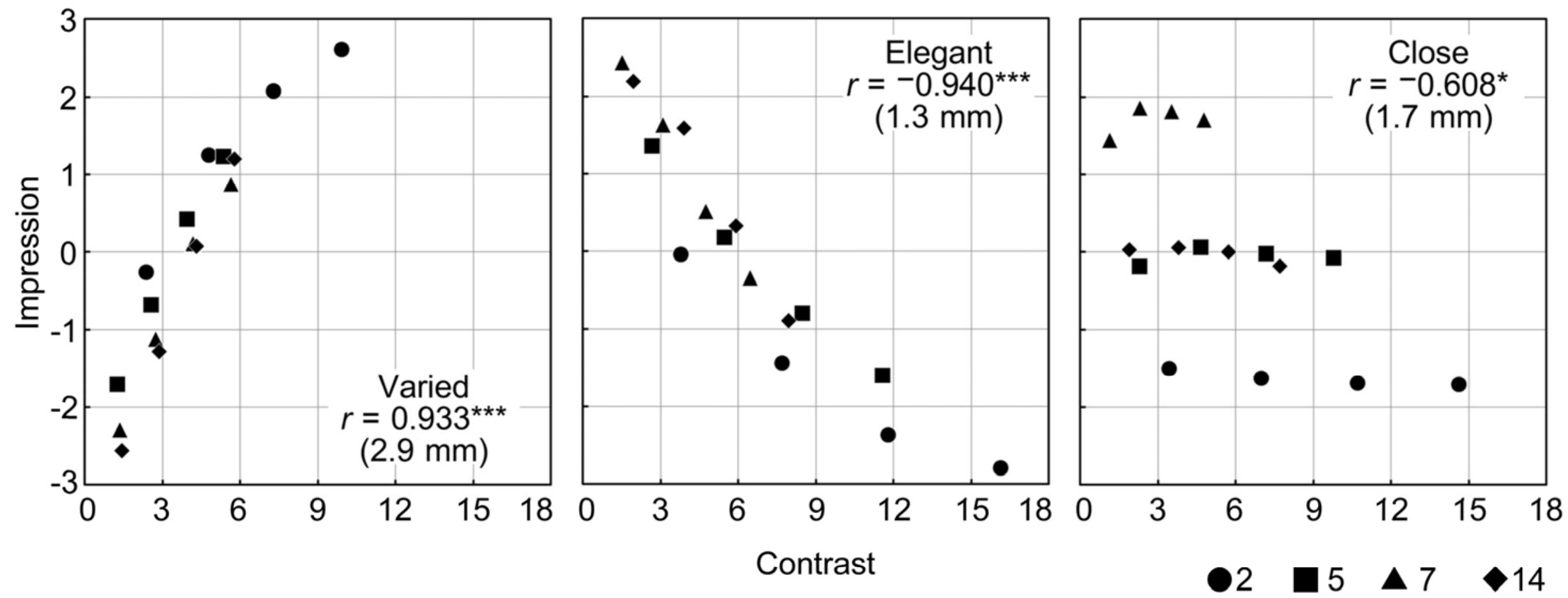

Fig. 6 


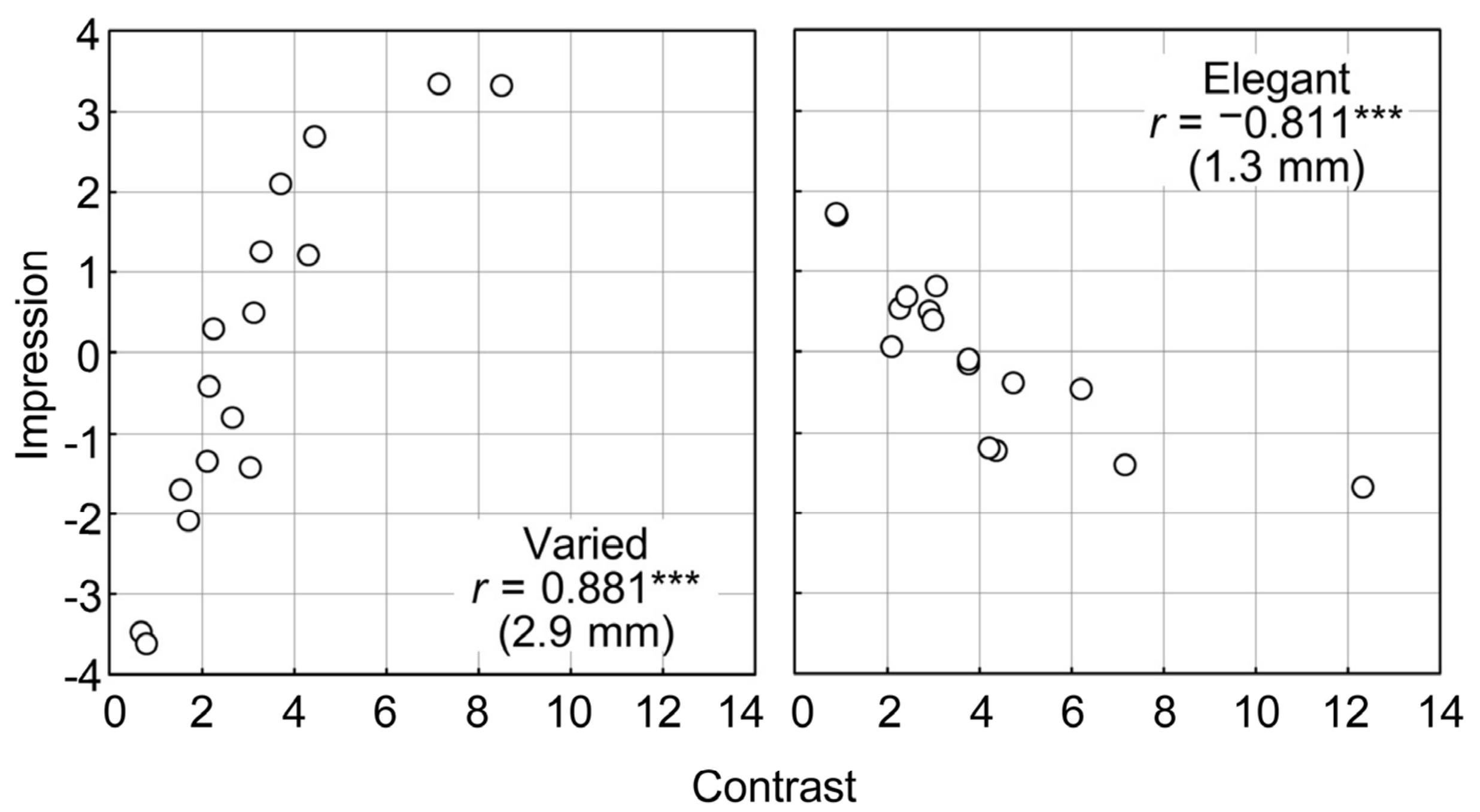

Fig. 7 


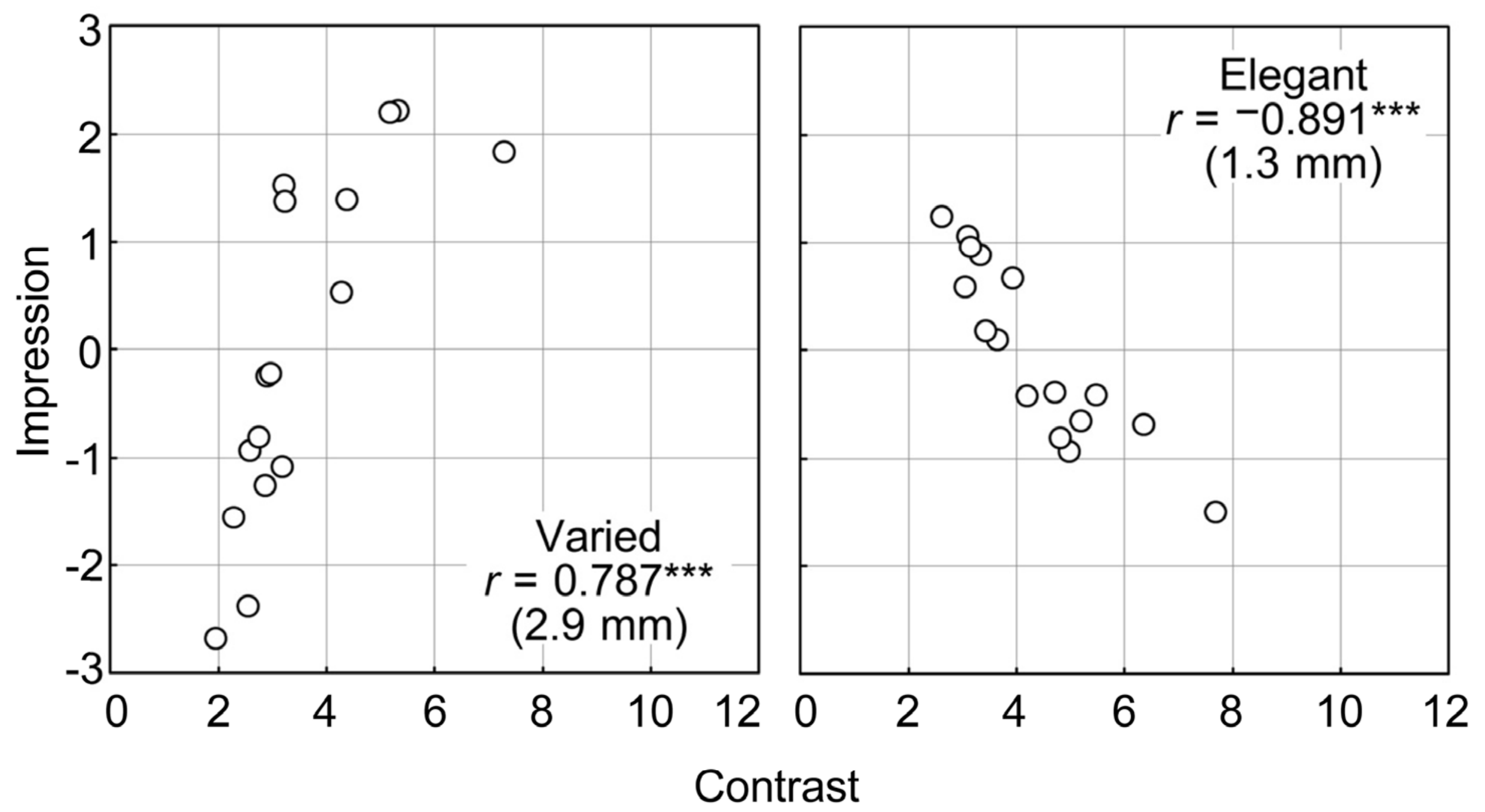

Fig. 8 

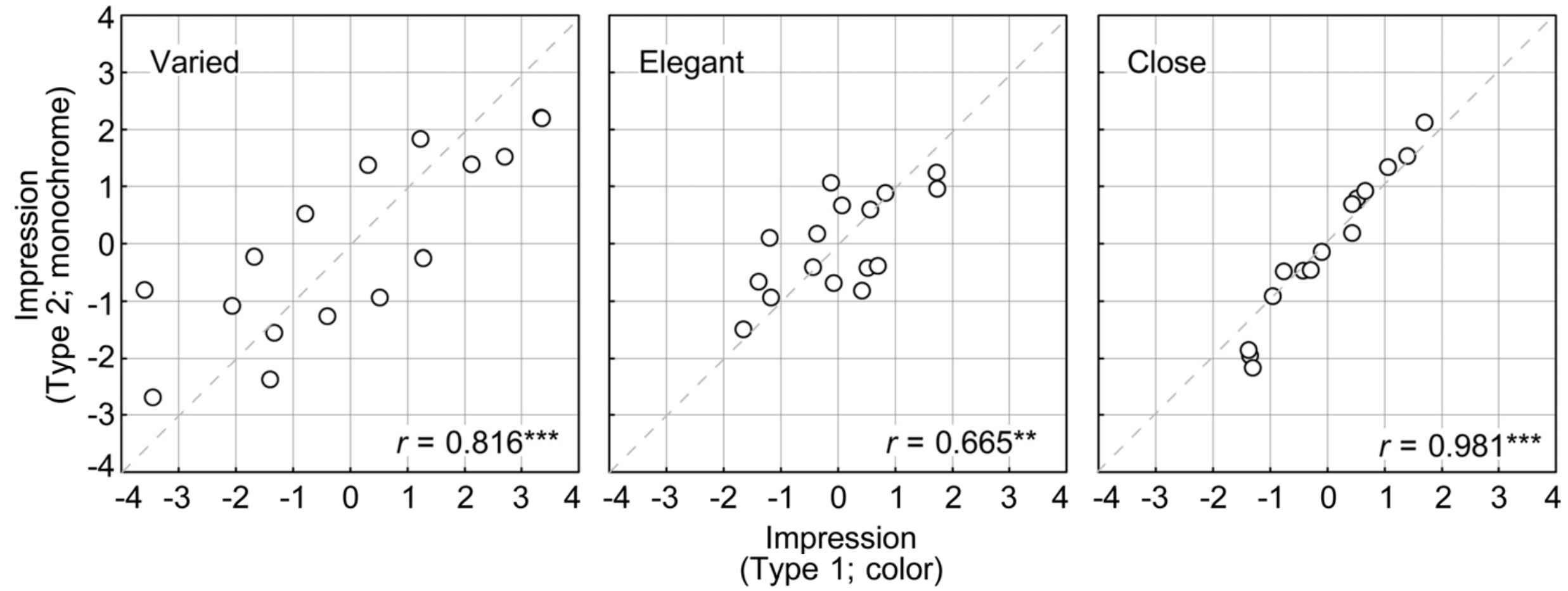

Fig. 9 
Table 1 Correlation matrix of impressions (Type 3)

\begin{tabular}{rlllll}
\hline & Close & Decent & Elegant & Clear & Varied \\
\hline Decent & $0.920 * * *$ & & & & \\
Elegant & $0.626 * *$ & 0.789 & & & \\
Clear & -0.210 & -0.372 & $-0.852 * * *$ & & \\
Varied & -0.482 & $-0.717 * * *$ & $-0.969 * * *$ & $0.857 * * *$ & \\
Showy & -0.462 & $-0.652^{* *}$ & $-0.973 * * *$ & $0.936 * * *$ & $0.968 * * *$ \\
\hline$* * p<0.01, * * * p<0.001$ & & & &
\end{tabular}

